\title{
A Fetal Heart Sound Signal De-Noising Approach Based on VMD and JADE Algorithm
}

\author{
ZhiweiNie \\ School of Computer Science, Guangdong University of Technology, China. \\ nzw2010@126.com
}

\begin{abstract}
A de-nosing approach is needed to eliminate the maternal heart sound and internal noise in the acquired fetal heart sound signal, but blind separation algorithm, as a conventional approach, does not perform well in the de-noising of fetal heart sound. Hence, a new fetal heart sound de-noising approach based on variational mode decomposition (VMD). Resampling is conducted for original signal on the basis of fetal heart sound signal characteristics, and then VMD is performed to select the signal components for independent component analysis with original signal, so as to obtain the denoised fetal heart sound signal. As revealed in experimental results, this approach achieves better de-noising than the conventional methods. Hence, it is a favorable de-noising approach to reduce the noise mixed in the original fetal heart sound signal, so as to improve the computational accuracy of fetal heart rate and provide the prerequisite for effective fetal heart monitoring.
\end{abstract}

Key words: Fetal heart sound; variational mode decomposition; JADE algorithm; de-noising.

\section{INTRODUCTION}

Fetal heart sound signal processing is a major way to learn about the fetal condition inside uterus, while signal denoising is one of its important steps. Common fetal Doppler acquires the signal, which contains not only fetal heart sound but also some noises such as noise caused by fetal movement, maternal heart sound, maternal breath sound and digestive sound, and other noise caused by move of measuring sensor and external environment. Fetal heart sound consists of the first heart sound (S1) caused by closing of mitral valve and tricuspid valve, the second heart sound (S2) resulting from blockage of flood backflow thanks to closing of pulmonary valve, the third heart sound (S3) caused at the beginning of diastole, and the fourth heart sound (S4) appearing at the end of diastole. Due to the weakness of S3 and S4 signals, S1 and S2 signals are generally acquired [1]. However, S1 and S2 signals fluctuate with the cycle of pregnancy, making it very difficult to de-noise fetal heart sound signals [2].

At present, as the fetal heart sound signals acquired by fetal heart monitoring device contain noise, fetal heart sound signal de-noising can be regarded as a blind source signal separation problem. Normally, blind separation algorithm is employed for blind signal separation [3], but it does not effectively de-noise the complex nonlinear and non-stationary signal of noise-containing fetal heart sound [4]. For this reason, domestic and foreign scholars have carried out extensive studies on the fetal heart sound signal de-noising approach. Yang et al. [5] practiced short-time Fourier transform in the time-frequency domain analysis of fetal heart sound signal, and effectively identified how the frequency of signal varied with time, by converting one-dimensional time domain signal into two-dimensional time frequency signal and conducting the united analysis of time domain and frequency domain. Akhtar et al. [6] applied the independent component analysis on the obtained spectrum components, which improved the accuracy of denoising compared to the single application of blind separation algorithm. Boudraa et al. [7] put forward a method to obtain the de-noised fetal heart sound signal by recombination after empirical mode decomposition. Dragomiretskiy et al. [8] studied the application of variational mode decomposition in signal de-noising and proved its great effect in 
processing non-stationary signals. Kuzilek et al. [9] introduced the JADE algorithm as a new method for extracting source signal from observed signal.

Considering the existing methods, this paper puts forth a fetal heart sound signal de-noising approach based on VMD and JADE algorithm. A fetal heart sound signal receives VMD for time frequency analysis to obtain a set of components from decomposition. After that, these components are combined on the basis of the known characteristics of the fetal heart sound signal. At last, the combination of components and the source signal of fetal heart sound are taken as the input matrixes for blind separation with JADE algorithm on the basis of matrix joint diagonalization, so as to remove the noise. This paper explores the procedure for realizing the proposed approach, studies its application in fetal heart sound signal de-noising, and presents an experiment. The experimental results show that the proposed approach can satisfy the needs of fetal heart sound signal de-noising and create the conditions for the subsequent detection of fetal heart rate.

\section{FETAL HEART SOUND SIGNAL DE-NOISING APPROACH BASED ON VMD AND JADE ALGORITHM}

\section{Variational Mode Decomposition}

Variational mode decomposition (VMD), a signal time frequency analysis method put forth by Dragomiretskiy et al. [8], follows the basic idea that it can adaptively decompose a complex non-stationary multi-component signal into the sum of several preset components, which are separated from the input signal at a center frequency. It can effectively extract the strong signal of low frequency. Hence, VMD can be applied in time frequency analysis on the basis of fetal heart sound signal characteristics.

It is assumed that the original signal $\mathrm{x}(t)$ consists of multiple signals subject to a center frequency, and the bandwidth of each signal represents the degree of its variation from the center frequency. VMD intends to find $\mathrm{k}$ functions $u_{k}$ and $w_{k}$ to minimize the sum of their bandwidths, i.e.

$$
\min _{\left\{u_{k}, w_{k}\right\}}\left\{\sum_{k}\left\|\partial_{t}\left[\left(\delta(t)+\frac{j}{\pi t}\right) * u_{k}(t)\right] e^{-j w_{k} t}\right\|_{2}^{2}\right\}
$$

At this time, there is:

$$
\mathrm{x}(t)=\sum_{k} u_{k}
$$

Given the threshold value $\varepsilon>0, \hat{u}_{k}^{n+1}(w), w_{k}^{n+1}$, and $\hat{\lambda}^{n+1}(w)$ are updated till:

$$
\sum_{k=1}^{K} \frac{\left\|\hat{u}_{k}^{n+1}(w)-\hat{u}_{k}^{n}(w)\right\|}{\left\|\hat{u}_{k}^{n}(w)\right\|_{2}^{2}}<\varepsilon
$$

With the above steps, k signal components with different center frequencies can be obtained from decomposition.

\section{JADE Algorithm}

JADE algorithm is a blind signal separation algorithm based on matrix joint diagonalization. In the separation process, it is necessary to conduct the independent component analysis of mixed signal, so there are very high requirements for the mixed signal as its input.

As shown in the above process, the JADE algorithm requires that the quantity of input data is not lower than the quantity of data to be separated. The fetal heart sound signal de-noising process is a mixed noise blind separation problem involving one input signal and multiple output signals, so data should be processed in the blind signal separation to satisfy the requirements for input. 


\section{Fetal Heart Sound Signal De-Noising Approach Based on VDM and JADE Algorithm}

Assuming that $\mathrm{x}(t)$ is the original signal, and the specific procedure of its de-noising is as follows:

(1) Pre-process the fetal heart sound signal on the basis of its characteristics to obtain the processed signal, i.e. $x_{1}(t)$

(2) Utilize the VMD algorithm to conduct the time frequency analysis on $x_{1}(t)$, and obtain $\mathrm{k}$ signal components;

(3) Select the signal components, mix them with simulated noise, and use them together with the original signal $x_{1}(t)$ as the input matrixes of the JADE algorithm

(4) Conduct the blind separation, calculate the separation signal, and select the noise signal.

(5) Remove it from the original signal to obtain the de-noised fetal heart sound signal.

On the basis of the above procedure, the process is presented as follows:

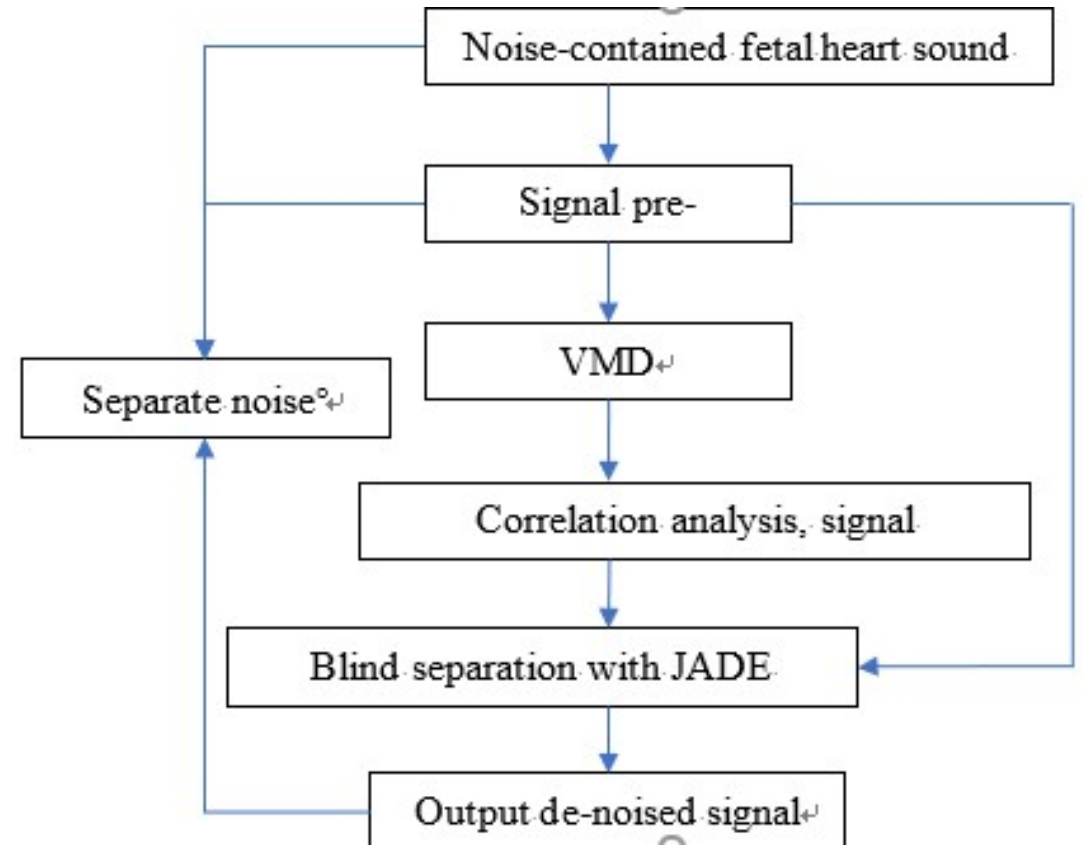

FIGURE 1. Process flow chart of fetal heart sound signal de-noising

\section{EXPERIMENT}

To verify the actual effectiveness of the proposed approach in fetal heart sound de-noising, the fetal heart sound signal provided by the MIT-BIH open database [10] is utilized. The experiment is entirely realized with the Windows 10 operating system and the Matlab for programming.

\section{Signal Pre-Processing}

In the MIT-BIH, the sampling rate of original signal for fetal heart sound is $1,000 \mathrm{~Hz}$, but the actual frequency of fetal heart sound is low in most cases [1]. Hence, a low-pass filter is designed as follows:

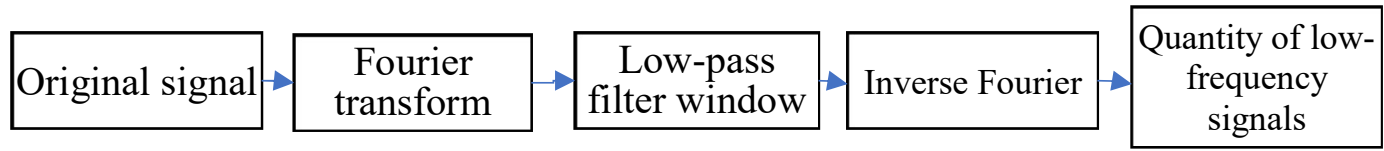

FIGURE 2. Process flow of filter processing 
Low-pass filter allows only the signals with frequency lower than cutoff frequency to pass and blocks all signals with frequency higher than cutoff frequency. Actually, this eliminates the high-frequency interferences in signal. As shown in the waveform and spectrogram of fetal heart sound electric signals, a large number of high-frequency signals exist particularly when the frequency is near $350 \mathrm{~Hz}$. According to records, the $\mathrm{S} 1$ and S2 signals needed in the analysis of fetal heart rate and other fetal analyses is normally not higher than $100 \mathrm{~Hz}$ [1]. Hence, the low-pass filter with the cutoff frequency set to $250 \mathrm{~Hz}$ is used.

After original signal passes the low-pass filter with the cutoff frequency of $250 \mathrm{~Hz}$, several crests are noticeable in its waveform graph, and the signal is also cyclical to some extent. As shown in the spectrogram, the frequency of signal is mainly $20 \mathrm{~Hz}$ and $60 \mathrm{~Hz}$, which matches with the record of S1 and S2 electric signal frequencies in Reference [1]. At this time, it is observed that fetal heart sound has two crests within less than $1 \mathrm{~s}$, which also matches with the record that fetal heart rate is $120-160 \mathrm{bpm}$.
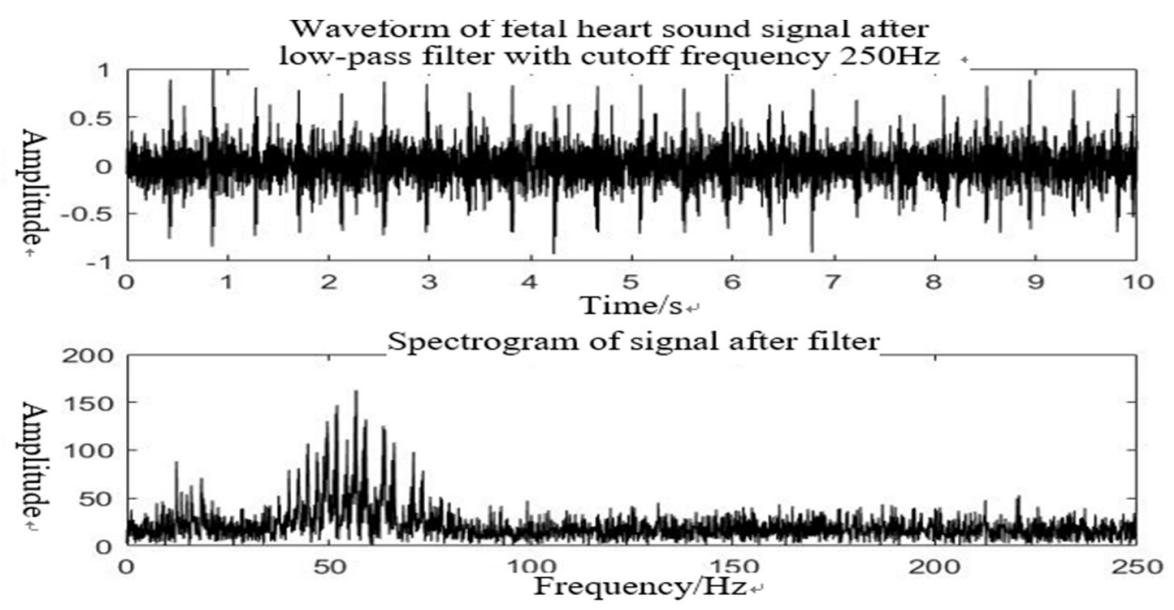

FIGURE 3. Waveform and spectrum of signal after low-pass filter

\section{Fetal Heart Sound Signal Time Frequency Analysis Based on VMD}

Following the procedure of VMD, the fetal heart sound signal after low-pass filter is decomposed. Considering the fetal heart sound signal characteristics [11], it is known that decomposition signal 1 and decomposition signal 2 are the components of the mixed signal of the effective fetal heart sounds S1 and S2, while decomposition signals 3, 4 and 5 are noise signals. On this basis, signals are combined to simulate noise signals:

$$
\mathrm{x}(t)=n(t)+f(t)
$$

In which, $\mathrm{x}(t)$ is the observed fetal heart sound signal, while $n(t)$ is the simulated noise signal. At this time, $\mathrm{K}$ signals are obtained after decomposition. In the experiment, $\mathrm{K}=5$.

\section{Creation of Simulated Noise Signal}

According to the principles of VMD, the decomposition of high-frequency signal is not so effective as expected [12]. It is assumed that decomposition signals 3,4 and 5 are $n_{1}(t), n_{2}(t), n_{3}(t)$ respectively, so the simulated signal $\hat{n}(t)$ can be obtained with the following formula:

$$
\hat{n}(t)=\mathrm{A} *\left(n_{1}(t) * \alpha+n_{2}(t) * \beta+n_{3}(t) * \gamma\right)
$$

It satisfies $\alpha+\beta+\gamma=1$. 


\section{Fetal Heart Sound De-Noising Algorithm with Simulated Noise Based on JADE}

On the basis of Equation (5), several noise signals are simulated and linearly mixed.

The simulated signals and fetal heart sound signal after high-pass filter are taken as input matrixes. The JADE algorithm is used for separation, and the number of separation signals is set to 4 . Then 4 signal components after separation are obtained.
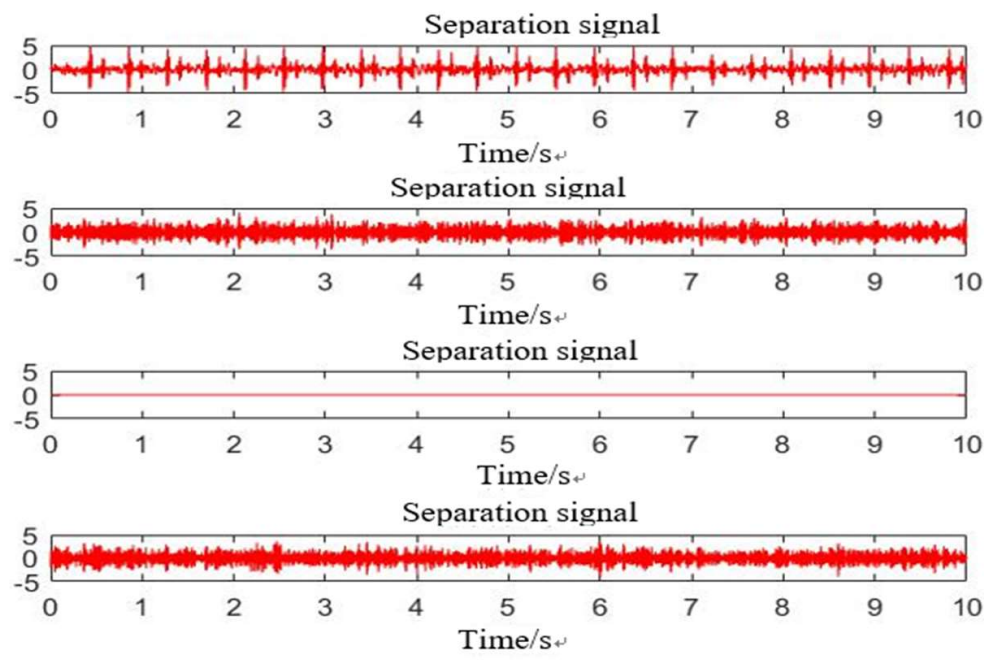

FIGURE 4. JADE blind separation signals

As shown in Fig. 9, after introducing 3 channels of simulated noise, the fetal heart sound signal mixed with noise is separated into 4 signals. The 4 output signals are analyzed, and separation signal 1 is selected to be approximate to the actual fetal heart sound signal. After that, the amplitude of the other 3 signal components is squeezed without changing the frequency characteristics. These signals are mixed according to the formula to obtain the mixing noise $\breve{\mathrm{n}}(t)$. Using the mixing noise in the linear computation together with the original signal $\mathrm{x}(t)$, the de-noised fetal heart sound signal $\hat{\mathrm{x}}(t)$ is obtained.
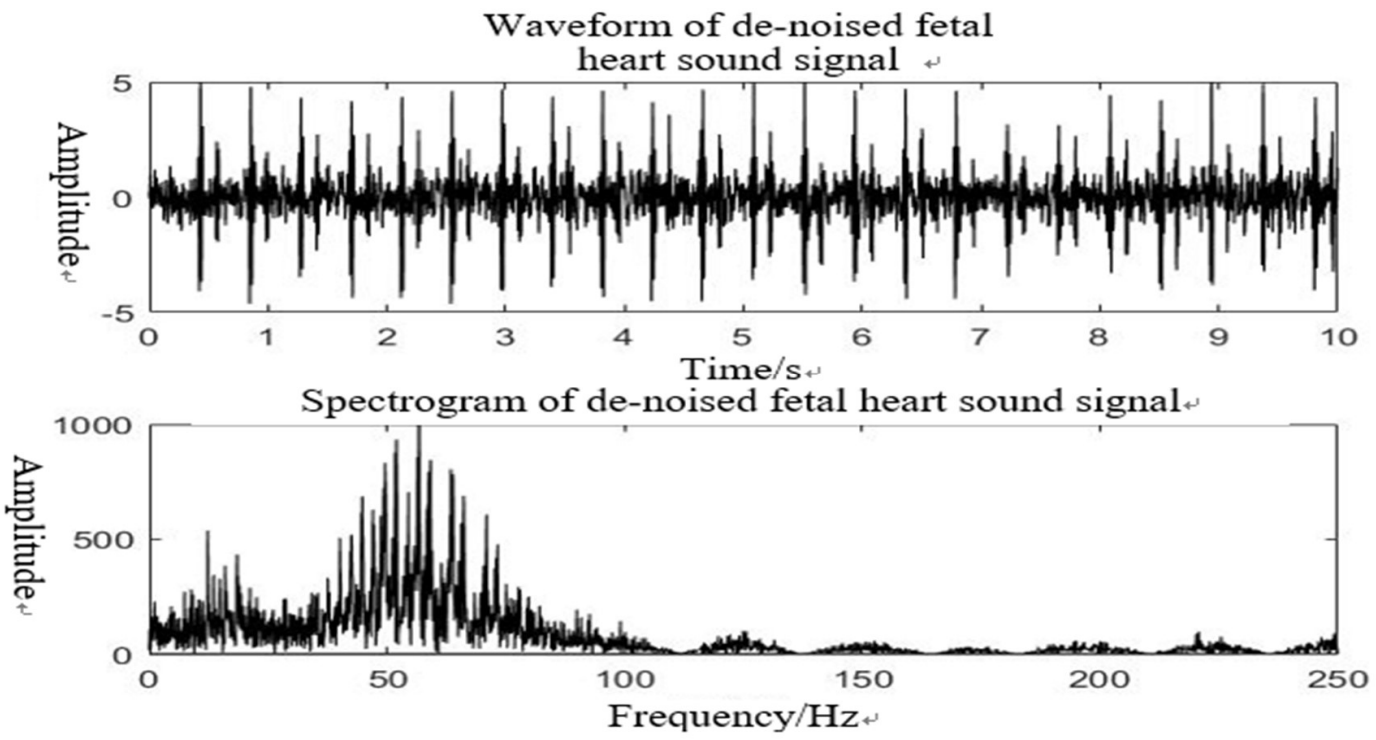

FIGURE 5. Waveform and spectrum of de-noised fetal heart sound signal 
As shown in Fig 5, the de-noised fetal heart sound signal has more noticeable crests in its waveform graph, and clearer spectrogram, so it can better reflect the regularity of fetal heart movement. It is therefore believed that the proposed approach reduces the proportion of noise in the fetal heart sound signal.

\section{ANALYSIS OF RESULTS}

To verify the effect of de-noising, the proposed approach is compared with other conventional methods. Signalto-noise ratio (SNR) is often used to evaluate the effect of de-noising [13]. SNR is measured in dB, and calculated with the following formula:

$$
\mathrm{SNR}=10 \log _{10} \frac{P_{S}}{P_{n}}
$$

In which, $P_{S}$ is signal energy after de-noising, and $P_{n}$ is the ability to remove de-noised signal from original signal, i.e. energy for separation of noise signal.

\section{SNR}

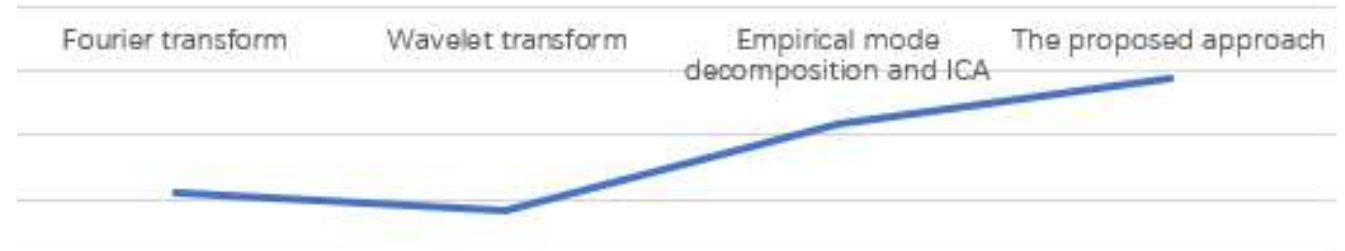

FIGURE 6. SNR with different de-noising methods

As shown in Fig. 11, the higher SNR, the better effect of de-noising [14]. It is clear that, if time frequency analysis is carried out alone for fetal heart sound signal, e.g. Fourier transform and wavelet transform, the effect of de-noising is inferior to the method of blind separation after signal decomposition. As shown in the SNR of the fetal heart sound signal, the proposed approach is better than the method of blind separation with independent component analysis (ICA) after empirical mode decomposition.

The proposed approach is employed to de-noise the fetal heart sound signals with different SNRs, which are selected from MIT-BIH, to obtain the de-noised signals of these source signals. After that, their SNRs are calculated with the formula.

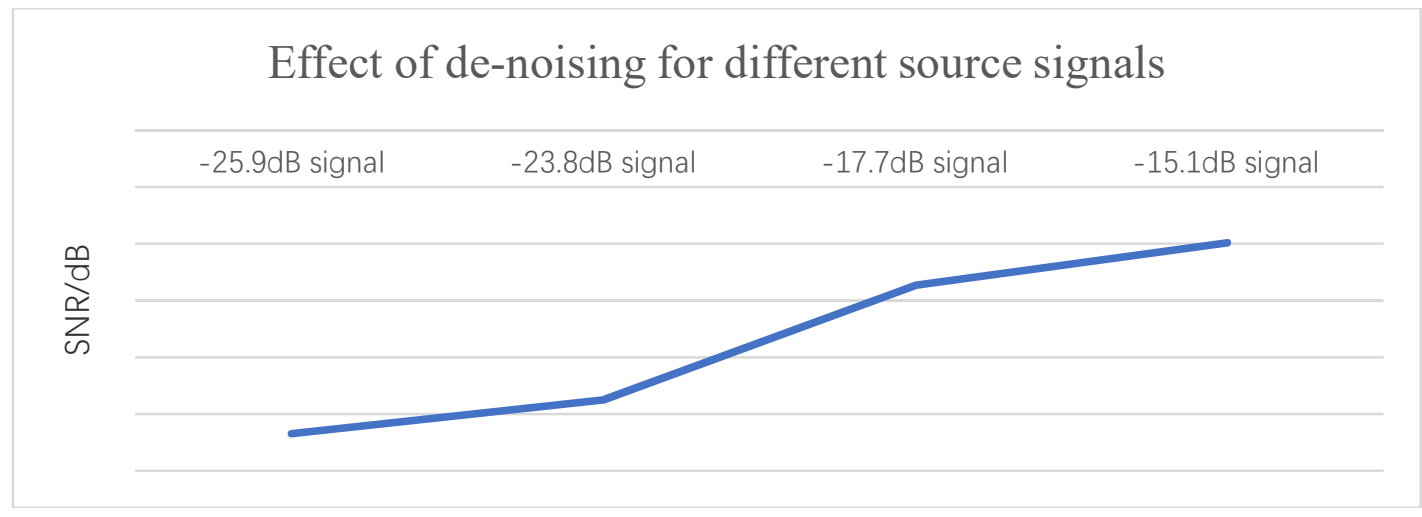

FIGURE 7. SNR of different input signals after de-noising

As shown in Fig. 12, when the SNR of original fetal heart sound signal is high, the SNR of the signal after denoising with the proposed approach is also high. Hence, the proposed approach has better effect of de-noising when 
the original fetal heart sound signal contains less noise. In practice, efforts should be made to avoid the noises caused by measuring device or external environment as much as possible.

\section{DISCUSSION}

This paper introduces a feasible approach to the de-noising problem of fetal heart sound signal, which successfully combines underdetermined blind separation with de-noising. In an experiment, the approach is applied in fetal heart sound signal, and it is found that the approach helps reduce the proportion of noise in the noise-contained fetal heart sound to some extent, so as to achieve the effect of de-noising. Meanwhile, the proposed approach is compared with other methods, and the results are analyzed to prove that the proposed approach has better effect in the fetal heart sound signal de-noising.

As an important task of fetal heart monitoring, fetal heart sound signal de-noising directly determines the quality of the results of the subsequent fetal heart sound analysis. The proposed approach can be taken as a method for data processing in the estimation of fetal heart rate. Through the estimation of fetal heart rate, it can further reflect the vital signs of fetus in mother's body, so as to diagnose early and cope with all kinds of unexpected conditions. The proposed approach presents a way to achieve fetal heart sound signal de-noising and can be also used in other data processing tasks requiring de-noising, e.g. mechanical failure diagnosis and traffic data de-noising.

Due to limited conditions, this paper verifies the proposed approach only with the data of fetal heart. Meanwhile, some parameters used in this paper should be further explored, e.g. VMD threshold value and number of decompositions. Meanwhile, fetal heart rate analysis, fetal heart abnormality monitoring and other tasks can be also carried out on this basis.

\section{REFERENCES}

1. Cesarelli M, Ruffo M, Romano M, et al. Simulation of foetal phonocardiographic recordings for testing of FHR extraction algorithms. [J]. Computer Methods \& Programs in Biomedicine, 2012, 107(3):513-523.

2. Kovács F, Török M, Habermajer I. A rule-based phonocardiographic method for long-term fetal heart rate monitoring[J]. IEEE transactions on bio-medical engineering, 2000, 47(1):124-30.

3. Cardoso J F. Blind signal separation: statistical principles[J]. Proc of IEEE, 1998, 86(10):2009-2025.

4. Kovács F, Török M, Habermajer I. A rule-based phonocardiographic method for long-term fetal heart rate monitoring[J]. IEEE transactions on bio-medical engineering, 2000, 47(1):124-30.

5. Yang X, Li P, Zhang X, et al. De-Noising of the Doppler Fetal Heart Rate Signal with Wavelet Threshold Filtering Based on Spatial Correlation[C]// The, International Conference on Bioinformatics and Biomedical Engineering. IEEE, 2007:928-931.

6. Akhtar M T, James C J. Focal artifact removal from ongoing EEG - a hybrid approach based on spatiallyconstrained ICA and wavelet de-noising[J]. Conf Proc IEEE Eng Med Biol Soc, 2009, 2009:4027-4030.

7. Boudraa A, Cexus J C. Denoising via empirical mode decomposition[J]. Proc IEEE Isccsp, 2006.

8. Dragomiretskiy K, Zosso D. Variational Mode Decomposition[J]. IEEE Transactions on Signal Processing, 2014, 62(3):531-544.

9. Kuzilek J, Kremen V, Lhotska L. Comparison of JADE and Canonical Correlation Analysis for ECG denoising[C]// Engineering in Medicine and Biology Society. IEEE, 2014:3857.

10. Goldberger AL, Amaral LAN, Glass L, Hausdorff JM, Ivanov PCh, Mark RG, Mietus JE, Moody GB, Peng CK, Stanley HE. PhysioBank, PhysioToolkit, and PhysioNet: Components of a New Research Resource for Complex Physiologic Signals. Circulation 101(23): e215-e220

11. Li Y N, Cai K. Detection of instantaneous heart rate of Doppler fetal heart sound signal based on independent component analysis [J]. China Medical Equipment Journal, 2006, 37(7):1-5.

12. Ma Z Q, Liu X Y, Zhang J J, et al. Application of VMD-ICA combined method in fault diagnosis of rolling bearings [J]. Journal of Vibration and Shock, 2017, 36(13):201-207.

13. Fujimori T, Miyazu T, Ishikawa K. Evaluation of analytical methods using signal-noise ratio as a statistical criterion [J]. Microchemical Journal, 1974, 19(1):74-85.

14. Wang J, Huang Q L. Research on the relationship of the signal- to- noise and the quantized value [J]. Spacecraft Recovery \& Remote Sensing, 2005, 26(2):25-28. 